\title{
Study on Hold
}

National Cancer Institute

\section{Source}

National Cancer Institute. Study on Hold. NCI Thesaurus. Code C70755.

A clinical study status designating that an order has been issued to a study sponsor to delay a proposed investig ation or to suspend an ongoing one. When a proposed study is placed on clinical hold, subjects may not be given the investigational product as part of the study. When an ongoing study is placed on clinical hold, no new subjects may be recruited to the study and placed on the investigational product. Subjects already in the study have to stop receiving therapy involving the investig ational agent unless the FDA specifically permits it. 